\title{
Atomic Electron Tomography: Probing 3D Structure and Material Properties at the Single-Atom Level
}

Yongsoo Yang ${ }^{1}$, Chien-Chun Chen ${ }^{1,2}$, M. C. Scott ${ }^{1,3}$, Colin Ophus ${ }^{3}$, Rui Xu ${ }^{1}$, Alan Pryor Jr. ${ }^{1}$, Li Wu ${ }^{1}$, Fan Sun ${ }^{4}$, Wolfgang Theis ${ }^{5}$, Jihan Zhou ${ }^{1}$, Markus Eisenbach ${ }^{6}$, Paul R. C. Kent ${ }^{7,8}$, Renat F. Sabirianov ${ }^{9}$, Hao Zeng ${ }^{4}$, Peter Ercius ${ }^{3}$ and Jianwei Miao ${ }^{1}$

1. Dept. of Physics and Astronomy and California NanoSystems Institute, UCLA, CA, USA.

2. Dept. of Physics, National Sun Yat-sen University, Kaohsiung, Taiwan.

3. NCEM, Molecular Foundry, Lawrence Berkeley National Laboratory, Berkeley, CA, USA.

4. Dept. of Physics, University at Buffalo, the State University of New York, Buffalo, NY, USA.

5. Nanoscale Physics Research Laboratory, School of Physics and Astronomy, University of

Birmingham, Edgbaston, Birmingham, UK.

6. National Center for Computational Sciences, ORNL, Oak Ridge, TN, USA.

7. Computer Science and Mathematics Division, ORNL, Oak Ridge, TN, USA.

${ }^{8 .}$ Center for Nanophase Materials Sciences, ORNL, Oak Ridge, TN, USA.

9. Dept. of Physics, University of Nebraska at Omaha, Omaha, NE, USA.

Perfect crystals are rare in nature. Many of the real materials contain crystal defects and chemical order/disorder including grain boundaries, dislocations, interfaces, surface reconstructions and point defects that disrupt the periodicity of the atomic arrangement and determine their properties and performance [1-3]. One prominent example is intermetallic compounds involving two or more atomic species, in which chemical order/disorder determines their mechanical, catalytic, optical, electronic and magnetic properties [4-6]. For instance, as-synthesized at room temperature, FePt nanoparticles and thin films with a near-1:1 composition have a chemically disordered fcc structure (A1 phase) [5,7,8]. When annealed at high temperatures, they undergo a transformation from an A1 phase to an ordered facecentered tetragonal $\left(\mathrm{L} 1_{0}\right)$ phase. Due to the chemical ordering and strong spin-orbit coupling [8], L10 FePt exhibits extremely large magnetocrystalline anisotropy energy (MAE) and is among the most promising candidates for next-generation magnetic storage media [7]. However, although this material system has attracted considerable attention, a fundamental understanding of 3D chemical order/disorder, crystal defects and its magnetic properties at the individual atomic level remains elusive.

Using atomic electron tomography (AET) [1,9-14], we determine the 3D coordinates of 6,569 iron and 16,627 platinum atoms in an FePt nanoparticle to correlate the 3D atomic structure with material properties at the single-atom level [15]. We identify rich structural variety and chemical order/disorder including 3D atomic composition, grain boundaries, anti-phase boundaries, anti-site point defects and swap defects. We show for the first time that experimentally measured 3D atomic coordinates and chemical species with $22 \mathrm{pm}$ precision can be used as direct input for first-principles calculations of material properties such as atomic magnetic moments and local magnetocrystalline anisotropy. This work not only opens the door to determining 3D atomic arrangements and chemical order/disorder of a wide range of nanostructured materials with high precision, but also will transform our understanding of structure-property relationships at a fundamental level [16].

References:

[1] J. Miao, P. Ercius and S. J. L. Billinge, Science 353 (2016), aaf2157.

[2] D. J. Bacon and D. Hull eds, "Introduction to Dislocations" 5th edn., (Butterworth-Heinemann, 
Oxford, 2011).

[3] A. A. Kelly and K. M. Knowles, "Crystallography and crystal defects" 2nd edn., (John Wiley \& Sons, Ltd., 2012).

[4] R. Ferrando et al, Chem. Rev. 108 (2008), pp. 845-910.

[5] S. Sun et al, Science 287 (2000), pp. 1989-1992.

[6] B. Lim et al, Science 324 (2009), pp. 1302-1305.

[7] G. Ju et al, IEEE Trans. Magn. (2015) 51, 3201709.

[8] B. D. Cullity and C. D. Graham, "Introduction to Magnetic Materials" 2nd edn., (Wiley, 2008).

[9] M. C. Scott et al, Nature 483 (2012), pp. 444-447.

[10] C.-C. Chen et al, Nature 496 (2013), pp. 74-77.

[11] R. Xu et al, Nat. Mater. 14 (2015), p. pp. 1099-1103.

[12] J. Park et al, Science 349 (2015), pp. 290-295.

[13] B. Goris et al, Nano Lett. 15 (2015), pp. 6996-7001.

[14] G. Haberfehlner et al, Nature Commun. 6 (2015), 8779.

[15] Y. Yang et al, Nature 542 (2017), pp. 75-79.

[16] This work was primarily supported by the US DOE (DE-SC0010378). This work was also supported by the US NSF (DMR-1548924 and DMR-1437263) and DARPA (DARPA-BAA-12-63). ADF-STEM imaging with TEAM I were performed at the Molecular Foundry, LBNL, which is supported by the US DOE (DEAC02-05CH11231). DFT calculations were supported by the US DOE. (DE-AC05-00OR22725).

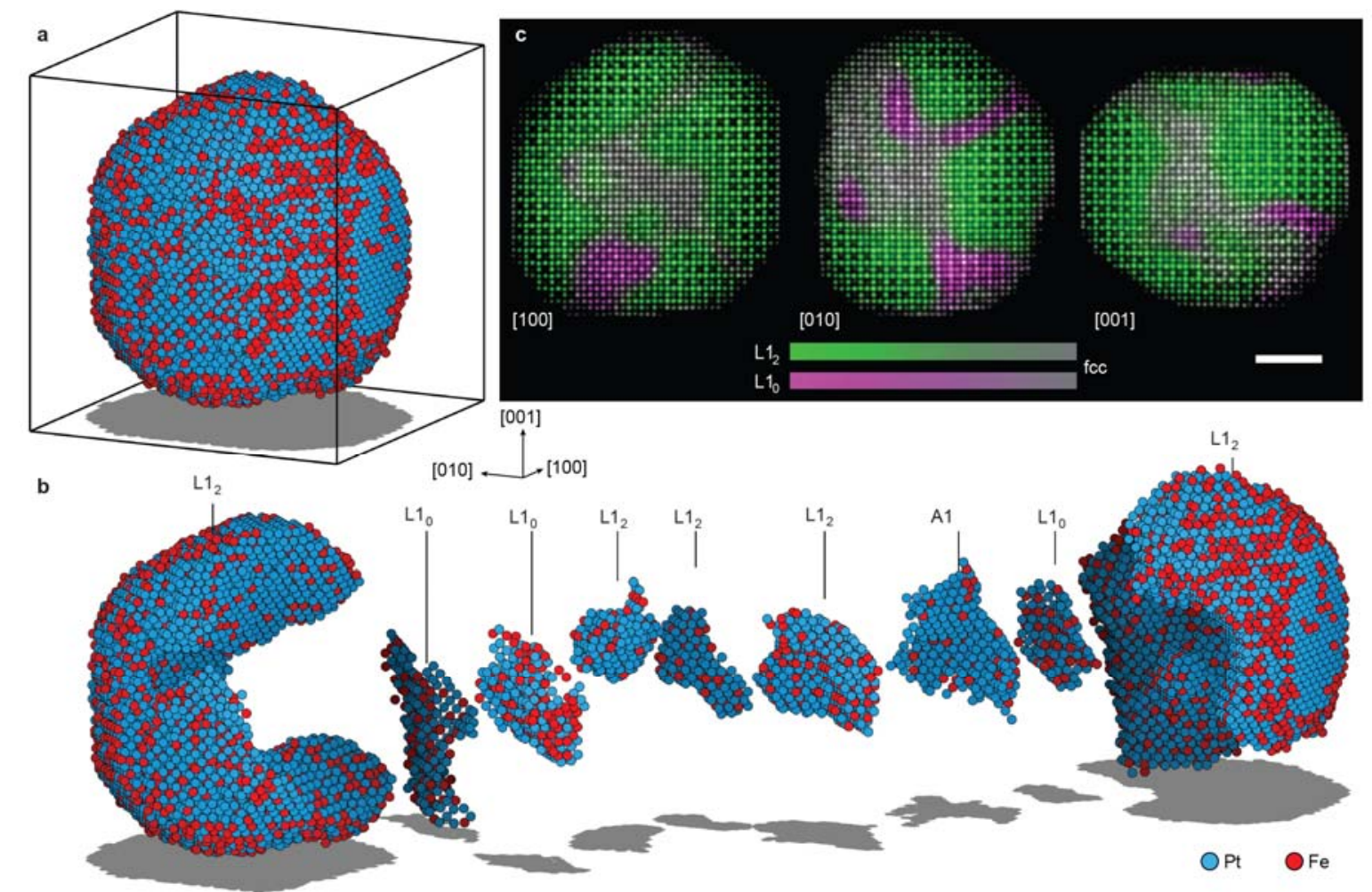

Figure 1. 3D determination of atomic coordinates, chemical species and grain structure of an FePt nanoparticle. a, Overview of the 3D positions of individual atomic species with Fe atoms in red and $\mathrm{Pt}$ atoms in blue. b. The nanoparticle consists of two large L12 grains, three small L12 grains, three small L10 grains and a Pt-rich A1 grain. c, Multislice images obtained from the experimental 3D atomic model along the [100], [010] and [001] directions, where several 'L10 grains' (magenta) appearing in the 2D images are deceptive structural information. Scale bar, $2 \mathrm{~nm}$. [Ref. 15] 